\title{
Deep Learning-Based Functional Independence Measure Score Prediction After Stroke in Kaifukuki (Convalescent) Rehabilitation Ward Annexed to Acute Care Hospital
}

Masahito Katsuki ${ }^{1,2}$, Norio Narita ${ }^{1}$, Dan Ozaki ${ }^{1}$, Yoshimichi Sato ${ }^{1}$, Wenting Jia $^{1}$, Taketo Nishizawa ${ }^{1}$ Ryuzaburo Kochi ${ }^{1}$, Kanako Sato ${ }^{1}$, Kokoro Kawamura ${ }^{1}$, Naoya Ishida ${ }^{1}$, Ohmi Watanabe ${ }^{1}$, Siqi Cai ${ }^{1}$, Shinya Shimabukuro ${ }^{1}$, Iori Yasuda ${ }^{1}$, Kengo Kinjo ${ }^{1}$, Kenichi Yokota ${ }^{1}$

1. Neurosurgery, Kesennuma City Hospital, Kesennuma, JPN 2. Neurosurgery, Itoigawa General Hospital, Itoigawa, JPN

Corresponding author: Norio Narita, naritanorio1111@gmail.com

\section{Abstract}

\section{Introduction}

Prediction models of functional independent measure (FIM) score after kaifukuki (convalescent) rehabilitation ward (KRW) are needed to decide the treatment strategies and save medical resources. Statistical models were reported, but their accuracies were not satisfactory. We made such prediction models using the deep learning (DL) framework, Prediction One (Sony Network Communications Inc., Tokyo, Japan).

\section{Methods}

Of the 559 consecutive stroke patients, 122 patients were transferred to our KRW. We divided our 122 patients' data randomly into halves of training and validation datasets. Prediction One made three prediction models from the training dataset using (1) variables at the acute care ward admission, (2) those at the KRW admission, and (3) those combined (1) and (2). The models' determination coefficients $\left(\mathrm{R}^{2}\right)$, correlation coefficients (rs), and residuals were calculated using the validation dataset.

\section{Results}

Of the 122 patients, the median age was 71, length of stay (LOS) in acute care ward 23 (17-30) days, LOS in KRW 53 days, total FIM scores at the admission of KRW 85, those at discharge 108. The mean FIM gain and FIM efficiency were 19 and 0.417. All patients were discharged home. Model (1), (2), and (3)'s R ${ }^{2}$ were 0.794, 0.970 , and 0.972 . Their mean residuals between the predicted and actual total FIM scores were $-1.56 \pm 24.6$, $4.49 \pm 17.1$, and $-2.69 \pm 15.7$.

\section{Conclusion}

Review began 06/29/2021 Review ended 07/09/2021 Published 07/23/2021

๑) Copyright 2021

Katsuki et al. This is an open access article distributed under the terms of the Creative Commons Attribution License CC-BY 4.0., which permits unrestricted use, distribution, and reproduction in any medium, provided the original author and source are credited.
Our FIM gain and efficiency were better than national averages of FIM gain 17.1 and FIM efficiency 0.187. We made DL-based total FIM score prediction models, and their accuracies were superior to those of previous statistically calculated ones. The DL-based FIM score prediction models would save medical costs and perform efficient stroke and rehabilitation medicine.

Categories: Neurology, Neurosurgery, Public Health

Keywords: artificial intelligence (ai), deep learning (dl), functional independent measure (fim), kaifukuki (convalescent) rehabilitation ward (krw), stroke, prediction model, prediction one, sony network communications inc. japan

\section{Introduction}

In Japan, stroke is the third leading cause of death and the second leading cause of long-term disability. Japan started a characteristic rehabilitation system in 2000 called kaifukuki (convalescent) rehabilitation wards (KRWs). KRW is incorporated into the Japanese medical insurance system, and The Japan Ministry of Health, Labour, and Welfare define KRWs as the essential inpatient rehabilitation system. Stroke patients are eligible for the KRW. They can undergo rehabilitation up to 150 days and 3 hours per day of rehabilitation, including physical, occupational, and speech therapy, in the KRW. There are over 60 KRW beds per 100,000 individuals, comprising $4.6 \%$ of the total Japanese hospital beds, and the number of KRW is increasing [1].

According to the Japanese Guidelines for the Management of Stroke 2015, rehabilitation should be performed to predict functional prognosis, hospital stay, and outcome-based activities of daily living, functional disability, and comorbidities [2]. Also, the KRW's quality should be maintained, and we should save the limited medical resources. Since 2017, the functional independence measure (FIM) score [3] has 
been used as a quality indicator of KRW, and hospitals with KRWs must achieve certain FIM-related standards [1]. Therefore, we try to increase and predict the total FIM score, FIM gain, and FIM efficiency (FIM gain divided by length of stay (LOS) in KRW) of each patient. Previously, several reports used multiple regression to predict total FIM score [4-15], but there are some questionable points; (1) not doing validation, (2) not confirming the normal distribution of variables and residuals to use multiple regression [16].

Generally, statistically making a prediction model needs many samples over thousands, so these studies tend to be country-initiated or academic association-initiated research. However, the larger the sample size, the less detailed information is available, such as comorbidities or laboratory test data, and the more there are missing data. Also, the treatment strategies vary from hospital to hospital, and patient backgrounds differ depending on countries and regions. Therefore, statistically making a universal prediction model for the FIM score is very difficult [16].

Recently, deep learning (DL), contained in artificial intelligence, is attractive. DL is gradually starting to be used in neurosurgical diseases in decision making for spinal canal stenosis [17], predicting outcomes after stroke [18,19], predicting the occurrence of stroke from meteorological information [20], automated diagnosis of primary headaches from a Japanese medical questionnaire [21], pathological diagnosis [22], or radiomics studies of brain tumours [23]. However, there are no reports on the DL-based prediction model regarding the total FIM score after KRW admission. We hypothesized that we could make a good prediction model for our hospital using the DL framework, even with a small dataset. Therefore, we herein produced the prediction model using DL framework, Prediction One (Sony Network Communications Inc., Tokyo, Japan, https://predictionone.sony.biz/) [19-21,24,25] with our dataset and compared the utility of the DLbased model to previously-reported multiple regression models. We also investigated our patients' characteristics admitted to KRW because our hospital is unique in that KRW is annexed to an acute care hospital.

\section{Materials And Methods}

\section{Study population}

We retrospectively retrieved data from medical records of all the consecutive 559 stroke patients admitted between 2017 and 2019 and treated at our institution. The stroke diagnosis was based on the clinical history and the findings of computed tomography (CT) or magnetic resonance imaging.

General management of stroke, including cerebral infarction (CI), intracerebral haemorrhage (ICH), and subarachnoid haemorrhage (SAH), was according to the Japanese Guidelines for the Management of Stroke 2015 [2]. We first performed acute care, including appropriate medical treatment and surgery. Rehabilitation is limited to $60 \mathrm{~min}$ in the acute phase. After 1-2 weeks of acute-phase rehabilitation, we discussed the patients' final destination with their families; home, KRW, nursing facilities, or long term hospitals. Patients who could live independently or with sufficient support from their families were discharged home in 2-4 weeks. Patients who needed more rehabilitation and might have the potential to live independently or with families' supports could be transferred to KRW. Patients who would not live independently or bed-ridden but whose families committed to providing in-home care could also be transferred to KRW, and such patients must have been discharged home. Patients who needed care and whose families could not support them were discharged to nursing facilities. Patients with severe neurological deficits like vegetative states were transferred to long term hospitals.

In the KRW, we could provide rehabilitation that was in line with real life. The daily rehabilitation was up to 3 hours, and the LOS was up to 150 days as maximum. We, doctors, nurses, and therapists, held monthly meetings with the patients and their families together, and we decided how long the rehabilitation in the KRW would be. This study retrospectively investigated the patients' characteristics, including age, stroke types, and discharge destination.

\section{Clinical variables for making prediction models}

We collected data regarding physiological symptoms at admission, i.e., date of admission, age, sex, height, weight, body mass index, history of smoking and habitual massive drinking (over 450g ethanol intake/week), comorbidities (history or present treatment by a clinician for hypertension, dyslipidemia, diabetes mellitus, chronic kidney diseases, orthopaedic diseases, or cancer). Glasgow Coma Scale (GCS) and National Institutes of Health Stroke Scale scores on admission were also investigated. We also measured triglycerides, total cholesterol, high-density lipoprotein cholesterol, low-density lipoprotein cholesterol, albumin, C-reactive protein, blood glucose, haemoglobin A1c, haemoglobin levels, and white blood cell and lymphocyte counts admission in the acute neurosurgical ward. Albumin, lymphocyte, and total cholesterol are known factors for controlling nutritional status scores to assess the patients' nutritional status [26]. As radiological findings, we investigated temporal muscle thickness (TMT) $(\mathrm{mm})$ as an indicator of systemic skeletal muscle mass [2734] based on the results of the CT at admission. SYNAPSE V 4.1.5 imaging software (Fujifilm Medical, Tokyo, Japan) was used through the methods described previously $[32,33]$.

We also investigated the modified Rankin Scale scores when the patients were transferred to the KRW. The 
LOS in the acute care ward and KRW were also investigated. Barthel Index (BI) at the admission of acute care ward, FIM score at admission to KRW and the discharge of KRW, FIM gain, and FIM efficiency were also collected, and the total FIM score at discharge was defined as an outcome in this study. There were no missing values.

\section{Making prediction model by Prediction One}

We used Prediction One framework to make the prediction models. We divided our 122 patients' data randomly into 61 patients training dataset and 61 patients validation dataset. Prediction One read the training data and automatically performed 5-folds cross-validation. Prediction One automatically adjusted and optimized the easy to process variables statistically and mathematically and select appropriate algorithms with ensemble learning. Prediction One made the best prediction model by an artificial neural network with internal cross-validation. The details are trade secrets and could not be provided.

We let the Prediction One framework make three prediction models using training dataset; (1) using 49 variables acquired only at the admission of acute care ward, (2) using 40 variables, including FIM scores, which could be known only at the admission of KRW, and (3) using all 70 variables acquired both at the admissions of acute care and KRW. The determination coefficient $\left(\mathrm{R}^{2}\right)$ and strong variables of each model were automatically calculated. Then, we performed validation using the validation datasets. Correlation coefficients (r) and residuals between the predicted and actual total FIM scores were calculated to evaluate the models' accuracy.

\section{Statistical analysis}

Results are basically shown as median (interquartile range). The difference between the training dataset and the external validation dataset was tested appropriately using the Mann-Whitney U test, Fisher's exact test, or Pearson's chi-square test. Univariate analysis on the association between each variable and total FIM score at the discharge of KRW was also performed. We could not perform multiple regression due to the small sample size and non-normal distribution of variables. A two-tailed $\mathrm{p}<0.05$ was considered statistically significant. We calculated $r$ and these $p$ values using SPSS software version 24.0.0. (IBM, New York, USA).

\section{Results}

\section{Clinical characteristics}

The clinical characteristics of the 559 stroke patients (421 CI, 98 ICH, 40 SAH; 224 women and 335 men) are summarized in table 1 . The median (interquartile range) age was 77 (69-83), and LOS in the acute care ward 15 (7-26). Regarding discharge destinations, 205 patients were discharged home, 122 patients were transferred to KRW, 81 nursing facilities, 79 long-term hospitals, and 72 patients died. 


\section{Cureus}

\begin{tabular}{|c|c|c|c|c|}
\hline & All stroke $(n=559)$ & $\mathrm{Cl}(\mathrm{n}=421,75 \%)$ & $\mathrm{ICH}(\mathrm{n}=\mathbf{9 8}, \mathbf{1 8} \%)$ & SAH $(n=40,7 \%)$ \\
\hline Age (median, interquartile range) & $77(69-83)$ & $77(70-83)$ & $79(69-83)$ & $66(59-83)$ \\
\hline $32-50$ y.o. & $17(3 \%)$ & $10(2 \%)$ & $5(5 \%)$ & $2(5 \%)$ \\
\hline $51-65$ у.о. & $68(12 \%)$ & $39(9 \%)$ & $13(13 \%)$ & $16(40 \%)$ \\
\hline 66-75 у.o. & $165(30 \%)$ & $139(33 \%)$ & $17(18 \%)$ & $9(21 \%)$ \\
\hline 76-85 y.o. & $224(40 \%)$ & $163(39 \%)$ & $51(52 \%)$ & $11(27 \%)$ \\
\hline $86-95$ y.o. & $79(14 \%)$ & 66 (16\%) & $12(12 \%)$ & $1(2 \%)$ \\
\hline 95- у.о. & $6(1 \%)$ & $4(1 \%)$ & 0 & $2(5 \%)$ \\
\hline Women: Men (Women\%) & 224: $335(40 \%)$ & 167: $254(40 \%)$ & 36: $62(37 \%)$ & 21: $19(53 \%)$ \\
\hline \multicolumn{5}{|l|}{ Discharge destination from the acute ward } \\
\hline Home & $205(37 \%)$ & $162(38 \%)$ & $32(33 \%)$ & $11(27 \%)$ \\
\hline KRW & $122(22 \%)$ & $73(17 \%)$ & $33(33 \%)$ & $17(43 \%)$ \\
\hline Nursing facility & $81(14 \%)$ & $62(15 \%)$ & $17(17 \%)$ & $2(5 \%)$ \\
\hline Long term hospital & $79(14 \%)$ & $64(16 \%)$ & $13(13 \%)$ & $2(5 \%)$ \\
\hline Dead & $72(13 \%)$ & $60(14 \%)$ & $4(4 \%)$ & $8(20 \%)$ \\
\hline LOS in an acute ward (days) (median, interquartile r & $15(7-26)$ & $19(7-45)$ & $21(12-44)$ & $10(1-39)$ \\
\hline
\end{tabular}

\section{TABLE 1: Characteristics of all stroke patients}

KRW; kaifukuki (convalescent) rehabilitation ward, LOS; length of stay.

Table 2 shows the treatment detail of 122 patients (67 CI, 42 ICH, and 13 SAH) who were transferred to KRW. Thirty-nine (32\%) of the 122 patients underwent aggressive treatment like intravenous tissue plasminogen activator, thrombectomy, hematoma removal surgery, or aneurysm ligation, rather than only medication.

\begin{tabular}{|c|c|c|c|c|c|}
\hline & Number & & Number & & Number \\
\hline Cl & 67 (5 t-PA, 5 t-PA + thrombectomy, 2 thrombectomy, 2 craniectomy) & $\mathrm{ICH}$ & 42 (9 surgery) & SAH & 13 \\
\hline ATB & 16 (3 t-PA, 1 craniectomy) & Putamen & 16 (5 surgery) & ACoA (clip) & 3 \\
\hline LI & $11(1 \mathrm{t}-\mathrm{PA})$ & Thalamus & 8 & ACD (clip) & 1 \\
\hline CE & 16 (5 t-PA + thrombectomy, 2 thrombectomy, 1 craniectomy) & Subcortex & 8 (3 surgery) & MCA (clip) & 4 \\
\hline BAD & 18 & Pons & 3 & ICA (clip) & 3 \\
\hline VAD & 3 & Cerebellum & 7 (1 surgery) & BA (coil) & 1 \\
\hline ESUS & 2 (1 t-PA) & & & VAD (coil) & 1 \\
\hline A-to-A & 1 & & & & \\
\hline
\end{tabular}

\section{TABLE 2: Stroke types and treatment of patients who underwent KRW}

ACoA; anterior communicating artery, ACD; distal part of anterior cerebral artery, A-to-A; artery to artery embolism, ATBI; atherothrombotic brain infarction, BA; basilar artery, BAD; brunch atheromatous disease, CE; cardiogenic embolism, Cl; cerebral infarction, ESUS; embolic stroke of undetermined sources, ICA; internal carotid artery, ICH; intracerebral hemorrhage, LI; lacunar infarction, MCA; middle cerebral artery, SAH; subarachnoid hemorrhage, t-PA; tissue-plasminogen activator; VAD; vertebral artery dissection.

Table 3 shows the characteristics of the 122 patients ( 49 women and 73 men). The median age was 71 (6479), LOS in acute care ward 23 (17-30) days, LOS in KRW 53 (35-92) days, total FIM scores at admission of 
KRW 85 (57-100), those at discharge 108 (86-118), FIM gain 19 (8-89), and FIM efficiency $0.271(0.147-$ $0.561)$. All 122 patients were discharged home. The mean FIM gain and FIM efficiency were 19 and 0.417 , although they were not with a normal distribution (Table 3). Age $(r=-0.487, p<0.001)$, LOS in KRW $(r=-$ $0.501, p<0.001)$, body weight $(r=0.322, p<0.001)$, GCS score at admission $(r=0.350, p<0.001)$, BI at admission of acute care ward $(r=0.435, p<0.001)$, total FIM score at admission of KRW $(r=0.874, p<0.001)$ were significantly associated with total FIM score at discharge of KRW in univariate analyses.

\begin{tabular}{|c|c|c|c|c|}
\hline & Total $(n=122)$ & $\mathrm{Cl}(\mathrm{n}=67)$ & $\mathrm{ICH}(\mathrm{n}=42)$ & $S A H(n=13)$ \\
\hline Age (median, interquartile range) & $71(64-79)$ & $53(35-92)$ & $70(65-78)$ & $65(57-75)$ \\
\hline Women: Men (Women\%) & 49:73 & 24:43 & 19:23 & 6:7 \\
\hline Body mass index $\left(\mathrm{kg} / \mathrm{m}^{2}\right)$ & $22.5(20.8-25.0)$ & $22.5(20.8-25)$ & $22.3(19.6-24.4)$ & $22.8(21.1-24.5)$ \\
\hline \multicolumn{5}{|l|}{ Past history } \\
\hline Presence of hypertension & $89(73 \%)$ & $46(69 \%)$ & $33(76 \%)$ & $10(77 \%)$ \\
\hline Presence of diabetes mellitus & $29(24 \%)$ & $19(28 \%)$ & $9(21 \%)$ & $1(8 \%)$ \\
\hline Presence of sydlipidemia & $30(26 \%)$ & $17(25 \%)$ & $7(17 \%)$ & $6(46 \%)$ \\
\hline History of smoking & $62(51 \%)$ & $35(52 \%)$ & $20(48 \%)$ & $7(54 \%)$ \\
\hline $\begin{array}{l}\text { History of massive alcohol } \\
\text { comsumption }\end{array}$ & $35(29 \%)$ & $21(31 \%)$ & $10(24 \%)$ & $4(31 \%)$ \\
\hline Presence of chronic kidney disease & $10(8 \%)$ & $5(7 \%)$ & $4(10 \%)$ & $1(8 \%)$ \\
\hline Presence of orthopedic disease & $15(12 \%)$ & $8(12 \%)$ & $6(14 \%)$ & $1(8 \%)$ \\
\hline Presence of malignant tumor & $7(6 \%)$ & $5(7 \%)$ & 0 & $2(15 \%)$ \\
\hline Glasgow Coma Scale on admission & $14(14-15)$ & $15(14-15)$ & $14(13-14)$ & $14(10-15)$ \\
\hline NIHSS on admission & $5(3-11)$ & $5(3-10)$ & $8(3-15)$ & - \\
\hline Barthel index on admission & $23(0-41)$ & $25(5-45)$ & $5(0-30)$ & $5(0-35)$ \\
\hline \multicolumn{5}{|l|}{ Laboratory test } \\
\hline Triglycerides (mg/dL) & $99(68-144)$ & $105(73-148)$ & $88(65-143)$ & $96(81-127)$ \\
\hline Total cholesterol (mg/dL) & $191(169-222)$ & $186(168-214)$ & $199(166-229)$ & $203(186-223)$ \\
\hline $\begin{array}{l}\text { High-density IIpoprotein cholesterol } \\
(\mathrm{mg} / \mathrm{dL})\end{array}$ & $49.9(42.5-68.9)$ & $48.1(42.2-64.0)$ & $48.6(45.5-75.0)$ & $71.0(48.1-75.0)$ \\
\hline $\begin{array}{l}\text { Low-density lipoprotein cholesterol } \\
(\mathrm{mg} / \mathrm{dL})\end{array}$ & $114(93-137)$ & $115(94-137)$ & $113(93-139)$ & $116(93-137)$ \\
\hline Albumin (g/dL) & $4.3(4.0-4.5)$ & $4.3(4.0-4.6)$ & $4.3(4.0-4.5)$ & $7.3(6.8-7.5)$ \\
\hline C-reactive protein (mg/dL) & $0.2(0.1-0.4)$ & $0.2(0.1-0.4)$ & $0.2(0.1-0.5)$ & $0.2(0.1-0.3)$ \\
\hline Blood glucose (mg/dL) & $133(108-165)$ & $130(106-168)$ & $133(112-159)$ & $144(122-163)$ \\
\hline HbA1c (\%) & $5.8(5.5-6.4)$ & $5.9(5.5-6.6)$ & $5.9(5.4-6.3)$ & $5.5(5.4-5.7)$ \\
\hline White blood cell count $(/ \mu \mathrm{L})$ & $7400(6000-9900)$ & $/ 150(5 / 50-8800)$ & $7 / 50(6000-10400)$ & $8800((2200-10300)$ \\
\hline Hemoglobin (g/dL) & $13.8(12.6-15.4)$ & $13.8(12.6-15.9)$ & $13.5(12.4-15.2)$ & $13.9(13.3-15.3)$ \\
\hline Lymphocyte count $(/ \mu \mathrm{L})$ & $1611(1198-2159)$ & $1570(1185-2025)$ & $1540(1309-2240)$ & $2083(1627-3313)$ \\
\hline Iemporal muscle thickness (mm) & $4.6(3.6-5.9)$ & $4.5(3.7-5.7)$ & $4.8(3.7-5.9)$ & $5.0(3.5-6.8)$ \\
\hline $\begin{array}{l}\text { Modified Rankin Scale when } \\
\text { transferred }\end{array}$ & $3(3-4)$ & $3(3-4)$ & $4(3-5)$ & $2(2-4)$ \\
\hline $\begin{array}{l}\text { LOS in an acute ward (days) } \\
\text { (median, interquartile range) }\end{array}$ & $23(17-30)$ & $23(17-29)$ & $22(17-29)$ & $30(26-33)$ \\
\hline FIM scores (median, interquartile & admission & admission & admission & admission \\
\hline
\end{tabular}




\section{Cureus}

\begin{tabular}{|c|c|c|c|c|c|c|c|c|}
\hline range) & to KRW & of KRW & to KRW & of KRW & to KRW & of KRW & to KRW & of KRW \\
\hline \multicolumn{9}{|l|}{ Self-care } \\
\hline Eating & $6(5-7)$ & $7(5-7)$ & $6(5-7)$ & $7(5-7)$ & $5(4-6)$ & $6(5-7)$ & $7(5-7)$ & $7(7-7)$ \\
\hline Grooming & $5(4-7)$ & $7(5-7)$ & $5(4-7)$ & $7(5-7)$ & $5(3-5)$ & $6(5-7)$ & $7(4-7)$ & $7(7-7)$ \\
\hline Bathing & $4(1-5)$ & $5(4-7)$ & $4(1-5)$ & $5(5-7)$ & $2(1-4)$ & $5(3-5)$ & $3(1-6)$ & $7(5-7)$ \\
\hline Dressing-Upper body & $5(2-7)$ & $7(5-7)$ & $5(4-7)$ & $7(6-7)$ & $4(2-6)$ & $6(4-7)$ & $6(3-7)$ & $7(7-7)$ \\
\hline Dressing-Lower body & $5(1-7)$ & $6(5-7)$ & $5(4-7)$ & $7(6-7)$ & $4(1-5)$ & $6(4-7)$ & $5(3-7)$ & $7(7-7)$ \\
\hline Toileting & $6(2-6)$ & $6(6-7)$ & $6(4-7)$ & $7(5-7)$ & $4(1-6)$ & $6(4-7)$ & $7(5-7)$ & $7(6-7)$ \\
\hline \multicolumn{9}{|l|}{ Sphincter Control } \\
\hline Bladder Management & $5(2-7)$ & $7(5-7)$ & $7(4-7)$ & $6(5-7)$ & $4(1-6)$ & $6(4-7)$ & $7(3-7)$ & $7(7-7)$ \\
\hline Bowel Management & $5(4-6)$ & $5(5-7)$ & $5(5-6)$ & $6(5-7)$ & $4(2-6)$ & $5(5-6)$ & $5(4-5)$ & $5(5-6)$ \\
\hline \multicolumn{9}{|l|}{ Transfer } \\
\hline Bed, Chair, Wheelchair & $5(3-7)$ & $6(5-7)$ & $5(5-7)$ & $6(6-7)$ & $5(2-6)$ & $5(5-7)$ & $7(4-7)$ & $7(7-7)$ \\
\hline Toileting & $5(3-6)$ & $6(5-7)$ & $6(5-7)$ & $5(4-7)$ & $4(2-5)$ & $6(5-7)$ & $7(4-7)$ & $7(7-7)$ \\
\hline Tub, Shower & $1(1-5)$ & $5(1-6)$ & $1(1-5)$ & $6(5-7)$ & $5(1-6)$ & $4(1-5)$ & $1(1-2)$ & $6(5-6)$ \\
\hline \multicolumn{9}{|l|}{ Locomotion } \\
\hline Walk/Wheelchair & $5(1-6)$ & $6(5-7)$ & $5(4-6)$ & $6(5-7)$ & $1(1-5)$ & $5(4-7)$ & $5(4-7)$ & $7(7-7)$ \\
\hline Stairs & $1(1-5)$ & $5(4-6)$ & $1(1-5)$ & $5(5-6)$ & $1(1-1)$ & $5(3-5)$ & $3(1-5)$ & $5(5-6)$ \\
\hline Motor Subtotal Score & $62(36-72)$ & $80(62-87)$ & $66(51-75)$ & $81(67-87)$ & $45(22-65)$ & $73(55-81)$ & $69(47-77)$ & $87(82-88)$ \\
\hline \multicolumn{9}{|l|}{ Communication } \\
\hline Comprehension & $5(3-6)$ & $6(5-7)$ & $5(4-6)$ & $6(5-7)$ & $5(3-6)$ & $6(5-7)$ & $5(2-7)$ & $7(5-7)$ \\
\hline Expression & $5(3-6)$ & $6(5-7)$ & $5(4-6)$ & $7(5-7)$ & $4(3-6)$ & $6(5-7)$ & $4(3-7)$ & $7(5-7)$ \\
\hline \multicolumn{9}{|l|}{ Social Cognition } \\
\hline Social Interaction & $6(4-6)$ & $7(6-7)$ & $6(5-6)$ & $7(6-7)$ & $5(4-6)$ & $6(6-7)$ & $5(3-6)$ & $7(6-7)$ \\
\hline Problem Solving & $4(2-5)$ & $5(3-6)$ & $5(2-5)$ & $5(4-6)$ & $2(2-6)$ & $5(2-5)$ & $5(2-5)$ & $5(5-7)$ \\
\hline Memory & $4(3-6)$ & $6(4-7)$ & $5(3-6)$ & $6(5-7)$ & $3(2-5)$ & $5(4-6)$ & $5(2-5)$ & $5(4-6)$ \\
\hline Cognitive Subtotal Score & $23(17-29)$ & $29(23-32)$ & $25(18-29)$ & $30(24-32)$ & $20(14-26)$ & $28(21-31)$ & $22(13-30)$ & $30(26-33)$ \\
\hline Total FIM Score & $85(57-100)$ & $\begin{array}{l}108(86- \\
118)\end{array}$ & $91(73-105)$ & $\begin{array}{l}111(89- \\
120)\end{array}$ & $70(44-89)$ & $97(72-109)$ & $97(83-103)$ & \\
\hline $\begin{array}{l}\text { LOS in KRW (days) (median, } \\
\text { interquartile range) }\end{array}$ & \multicolumn{2}{|l|}{$53(35-92)$} & \multicolumn{2}{|l|}{$47(35-68)$} & \multicolumn{2}{|l|}{$66(45-117)$} & \multicolumn{2}{|l|}{$36(32-67)$} \\
\hline FIM gain (median) & \multicolumn{2}{|l|}{19 (8-89) } & \multicolumn{2}{|l|}{$16(7-25)$} & \multicolumn{2}{|l|}{$25(15-31)$} & \multicolumn{2}{|l|}{$16(12-24)$} \\
\hline FIM efficiency (median) & \multicolumn{2}{|c|}{$0.271(0.147-0.561)$} & \multicolumn{2}{|c|}{$0.286(0.133-0.589)$} & \multicolumn{2}{|c|}{$0.254(0.146-0.423)$} & \multicolumn{2}{|c|}{$0.400(0.105-0.735)$} \\
\hline FIM gain $(m e a n \pm S D)$ & $19 \pm 19$ & & $16 \pm 11$ & & $23 \pm 17$ & & $16 \pm 12$ & \\
\hline FIM efficiency (mean $\pm S D)$ & $0.417 \pm 0.652$ & & $0.456 \pm 0.820$ & & $0.308 \pm 0.280$ & & $0.457 \pm 0.385$ & \\
\hline
\end{tabular}

\section{TABLE 3: Detailed characteristics of patients who were hospitalized in KRW}

$\mathrm{Cl}$; cerebral infarction, FIM; functional independent measure, $\mathrm{ICH}$; intracerebral hemorrhage, KRW; kaifukuki (convalescent) rehabilitation ward, LOS; length of stay, NIHSS; National Institutes of Health Stroke Scale, SAH; subarachnoid hemorrhage, SD; standard deviation. 
There were no significant differences in the variables between the training and validation datasets. Prediction One produced each prediction model in less than two minutes. The $\mathrm{R}^{2}$ of each model were described in Table 4. Model (1), using 49 variables acquired only at the acute care ward admission, had an $\mathrm{R}^{2}$ of 0.794 . Its $r$ and mean \pm standard deviation of residuals between the predicted and actual total FIM scores were 0.372 (95\%confident interval $0.120-0.578$ ) and $-1.56 \pm 24.6$. Model (2) using 40 variables acquired only at the admission of KRW had $\mathrm{R}^{2}$ of 0.970 , and its $\mathrm{r}$ and residuals were 0.789 (0.788-0.790) and $-4.49 \pm 17.1$.

Model (3) using all 70 variables acquired both in the acute and KRW had $\mathrm{R}^{2}$ of 0.974 , and its $\mathrm{r}$ and residuals were $0.810(0.810-0.811)$ and $-2.69 \pm 15.7$. The results are compared to those of previous reports [4-

15] (Table 4).

\begin{tabular}{|c|c|c|c|c|c|c|c|c|c|c|c|c|}
\hline Year & Author & $\begin{array}{l}\text { Number } \\
\text { of } \\
\text { variables }\end{array}$ & $\mathbf{R}^{2}$ & institutions & $\begin{array}{l}\text { Number } \\
\text { of } \\
\text { patients }\end{array}$ & $\begin{array}{l}\text { Mean } \\
\text { age }\end{array}$ & $\begin{array}{l}\text { Mean } \\
\text { LOS in } \\
\text { the acute } \\
\text { ward } \\
\text { (days) }\end{array}$ & $\begin{array}{l}\text { Mean } \\
\text { LOS in } \\
\text { the } \\
\text { KRW } \\
\text { (days) }\end{array}$ & Validation & $\begin{array}{l}\mathbf{R}^{2} \text { of } \\
\text { validation }\end{array}$ & $\begin{array}{l}r \text { of } \\
\text { validation }\end{array}$ & $\begin{array}{l}\text { mean } \\
\text { residual } \pm \text { SD }\end{array}$ \\
\hline 1997 & Liu [4] & 1 & 0.798 & single & 106 & 56.5 & 83 & 105.5 & - & & & \\
\hline 2000 & Tsuji [5] & 3 & 0.64 & multiple & $\begin{array}{l}190 \\
\text { training + } \\
116 \\
\text { validation }\end{array}$ & 61 & 47.3 & 90.9 & + & 0.68 & & \\
\hline 2001 & Inouye [6] & 1 & 0.57 & $\begin{array}{l}\text { single } \\
\text { (aged over } \\
80 \text { ) }\end{array}$ & 464 & 60 & 74 & 116 & - & & & \\
\hline 2001 & Inouye [6] & 4 & 0.76 & $\begin{array}{l}\text { single } \\
\text { (aged 60- } \\
69 \text { ) }\end{array}$ & 464 & 60 & 74 & 116 & - & & & \\
\hline 2005 & Sonoda [7] & 4 & $\begin{array}{l}\text { Not } \\
\text { described }\end{array}$ & single & $\begin{array}{l}87 \\
\text { training + } \\
44 \\
\text { validation }\end{array}$ & 63.4 & 81.3 & 154.3 & + & & 0.88 & $8.06 \pm 6.29$ \\
\hline 2016 & $\begin{array}{l}\text { Tsuchiya } \\
\text { [8] }\end{array}$ & 3 & 0.30 & single & 108 & 66.8 & 45.5 & 99.9 & - & & & \\
\hline 2016 & Senda [9] & 5 & 0.591 & single & 520 & 72.8 & 32.8 & 55.4 & - & & & \\
\hline 2016 & $\begin{array}{l}\text { Nishioka } \\
{[10]}\end{array}$ & 8 & 0.663 & single & 897 & 71.6 & $\begin{array}{l}21 \\
\text { (median) }\end{array}$ & $\begin{array}{l}122 \\
\text { (median) }\end{array}$ & - & & & \\
\hline 2017 & $\begin{array}{l}\text { Shiraishi } \\
\text { [11] }\end{array}$ & 3 & 0.776 & single & $\begin{array}{l}108 \\
\text { (including } \\
\text { not only } \\
\text { stroke } \\
\text { patients) }\end{array}$ & 80.5 & $\begin{array}{l}\text { Not } \\
\text { described }\end{array}$ & 65.2 & - & & & \\
\hline 2019 & $\begin{array}{l}\text { Matsushita } \\
\text { [12] }\end{array}$ & 3 & 0.673 & single & 267 & 72.5 & 24 & 105.1 & - & & & \\
\hline 2019 & $\begin{array}{l}\text { Nishioka } \\
\text { [13] }\end{array}$ & 6 & 0.742 & multiple & $\begin{array}{l}5549 \\
\text { (including } \\
\text { not only } \\
\text { stroke } \\
\text { patients) }\end{array}$ & 82 & $\begin{array}{l}\text { Within } 2 \\
\text { months. }\end{array}$ & 72 & - & & & \\
\hline 2019 & $\begin{array}{l}\text { Yoshimura } \\
\text { [14] }\end{array}$ & 6 & 0.719 & single & 276 & 74.9 & 15 & 99.9 & - & & & \\
\hline 2019 & Senda [15] & 2 & 0.691 & single & 371 & 72.9 & 31.4 & 79.7 & - & & & \\
\hline 2021 & Ours & 49 & 0.794 & single & $\begin{array}{l}61 \\
\text { training + } \\
61 \\
\text { validation }\end{array}$ & 69.4 & 24.3 & 53 & + & & $\begin{array}{l}0.372 \\
(0.120- \\
0.578)\end{array}$ & $-1.56 \pm 24.6$ \\
\hline
\end{tabular}




\section{Cureus}

\begin{tabular}{|c|c|c|c|c|c|c|c|c|c|c|c|}
\hline 2021 & Ours & 40 & 0.970 & single & $\begin{array}{l}61 \\
\text { training + } \\
61 \\
\text { validation }\end{array}$ & 69.4 & 24.3 & 53 & + & $\begin{array}{l}0.789 \\
(0.788- \\
0.790)\end{array}$ & $-4.49 \pm 17.1$ \\
\hline 2021 & Ours & 70 & 0.974 & single & $\begin{array}{l}61 \\
\text { training + } \\
61 \\
\text { validation }\end{array}$ & 69.4 & 24.3 & 53 & + & $\begin{array}{l}0.810 \\
(0.810- \\
0.811)\end{array}$ & $-2.69 \pm 15.7$ \\
\hline
\end{tabular}

\section{TABLE 4: Previous prediction models for total FIM score at discharge}

FIM; functional independent measure, KRW; kaifukuki (convalescent) rehabilitation ward, LOS; length of stay, r; correlation coefficients, R²; determination coefficients

The more robust variables of each model are listed in Table 5. In model (1), TMT, lymphocyte count, lowdensity lipoprotein cholesterol level, total BI score, and haemoglobin level had enormous effects on the outcome. In model (2), Age, FIM (bowel management), TMT, FIM (toileting), and LOS in the acute care ward were important. In model (3), Total FIM scores, subtotal cognitive FIM score, FIM (comprehension), subtotal motor FIM score, and blood glucose levels were meaningful, in order.

\begin{tabular}{|l|l|l|l|}
\hline Order & $\begin{array}{l}\text { Model (1) using } 49 \text { variables acquired only } \\
\text { at the admission of acute care ward }\end{array}$ & $\begin{array}{l}\text { Model (2) using } 40 \text { variables } \\
\text { acquired only at the admission of } \\
\text { KRW }\end{array}$ & $\begin{array}{l}\text { Model (3) using all 70 variables acquired at the } \\
\text { admission of both in acute care and KRWs }\end{array}$ \\
\hline 1 & Temporal muscle thickness & Age & Total FIM scores \\
\hline 2 & Lymphocyte & FIM bowel management & Subtotal cognitive FIM score \\
\hline 3 & Low-density lipoprotein cholesterol & Temporal muscle thickness & FIM comprehension \\
\hline 4 & Total Barthel Index score & FIM toileting & Subtotal motor FIM score \\
\hline 5 & Hemoglobin & LOS in the acute ward & Blood glucose \\
\hline 6 & NIHSS score & FIM dressing lower body & FIM bathing \\
\hline 7 & Blood glucose & FIM expression & Temporal muscle thickness \\
\hline 8 & Height & FIM stairs & Total cholesterol \\
\hline 9 & Total cholesterol & Height & Total BI at admission \\
\hline 10 & Bl (on level surfaces) & Subtotal cognitive FIM score & FIM problem solving \\
\hline
\end{tabular}

\section{TABLE 5: Stronger variables of each model provided by Prediction One (Sony Network} Communications Inc., Japan)

BI; Barthel index, FIM; functional independent measure, KRW; kaifukuki (convalescent) rehabilitation ward, LOS; length of stay, NIHSS; National Institutes of Health Stroke Scale

\section{Discussion}

We made prediction models for the total FIM score at the discharge of KRW in our hospital. We made three models; (1) using information gained at the admission of acute care ward, (2) using information gained at the admission of KRW, and (3) combined (1) and (2). DL-based models (2) and (3) had good accuracies, and we also performed validation, despite our small dataset. This is the first report on creating DL-based prediction models of total FIM scores at the discharge of KRW.

\section{KRW annexed to acute care hospital}

Our hospital was rebuilt in 2017, and KRW was newly annexed. Until then, patients had been transferred to the other hospitals' KRWs, but it took more than a month from the stroke onset to transfer. Since the new hospital, the transfer to our KRW from the stroke onset has been shortened to 23 days. Furthermore, the mean FIM gain of 19 and the mean FIM efficiency of 0.417 is higher than those of national averages of 17.1 
and 0.187 [35]. This may be because the staff in the acute care ward and those in KRW work closely together and frequently held meetings with patients and their families. Sharing the information on patients' comorbidities, treatment status, and rehabilitation progress will allow us to smooth transfer. This openness is one of the advantages of our hospital.

In our hospital, all patients were discharged home because we only permitted the transfer to KRW for patients who might have the potential to live independently with/without families' support or those who would not live independently or bed-ridden but whose families committed to providing in-home care. We decided on these determinations after around 1-2 weeks after onset. Whether it is socially and medically right to make such an early decision needs further discussion. However, we are forced to make such early decisions to use our limited medical resources in this rural area effectively. In this situation with limited medical resources, total FIM score prediction at the discharge of KRW is essential for the effective use of limited medical resources and the decision-making of patients and their families.

\section{Advantages of DL}

Conventional cost- and time-consuming statistical analysis needs variable optimization like a logarithmic transformation to make the variables with normal distribution increase the prediction model's accuracy mathematically. It also requires the arbitrary selection of variables based on previous studies, and multivariate analysis needs $10-15$ folds number of samples against the variables [36]. Therefore, there is a risk that variables that may be important cannot be used in the statistical analysis. Even the multivariate analysis cannot be done in a small hospital with small data. Furthermore, we should do multiple imputations or listwise deletion in statistical analysis when there is missing data, leading to inaccuracy. Furthermore, we should validate the models' accuracy, but several previous reports were not validated [16].

DL has the potential to overcome these problems [37]. DL develops useful models with less effort or time using the small dataset, without time-consuming variable optimization nor arbitrarily choosing variables because the DL framework automatically performs these processes. The optimal number of the variables for the DL framework is not revealed, and the DL framework sometimes finds interesting variables as necessary that has not been taken into account in the previously reported statistical models. Furthermore, the DL framework automatically uses appropriate values instead of the missing ones and calculate the best prediction model.

We then review these benefits of DL in our study. Conventionally, we could have used only six variables for statistical analysis due to the small sample size of the training dataset $(n=61)$. However, we could use 70 variables for making the model (3) and make a good prediction model from the small dataset. We did not need to perform variable optimization by ourselves. Furthermore, some unexpected variables, such as TMT, lymphocyte count, blood glucose level, were judged to be important in DL models. The suggestions are essential because data from the acute phase affects the total FIM score after KRW admission. What was not statistically significant in univariate analyses was deemed essential. Besides, the time needed for creating each model was less than 2 minutes. Finally, the models achieved high accuracies both in the training and validation dataset. While many reports did not conduct validation, we believe that our report is important, creating a stir about validation to present the accuracy.

\section{Limitations of DL}

First, the prediction model derived from our data cannot be applied to other institutions. The training and validation dataset must be updated to keep up with advances in medical science and surgical techniques, and medication changes. Creating a DL-based prediction model that can be used universally at any hospital will still require country-initiated or academic association-initiated collaborative research at many institutions. It may eventually require the same amount of effort as the traditional statistical model creation.

\section{Limitation of this study}

First, the sample size was small, and it is unknown how many samples are needed for DL analysis. We did not investigate the detail of rehabilitation training and how long the rehabilitation was actually performed per day for each individual. Further continuation study is needed.

\section{Conclusions}

Our hospital has the acute care ward and KRW. The FIM efficiency and LOS in the acute care ward were better than those in national averages. We easily and quickly made total FIM score prediction models using Prediction One framework. The accuracies of the prediction models were superior to those of previous statistically calculated prediction models. Even with a small single-centre dataset, DL-based total FIM score prediction models made by Prediction One can be helpful at the institution. They may be applied to daily clinical practice in the future.

\section{Additional Information}




\section{Disclosures}

Human subjects: Consent was obtained or waived by all participants in this study. Kesennuma City Hospital Ethics Committee issued approval 2020-1-5. The Kesennuma City Hospital's Research Ethics Committee approved this study, and the approval number is 2020-1-5. We gained written informed consent for this study from all of the patients, the legally authorized representative of the patients, or the next of kin of the deceased patients. All methods were carried out in accordance with relevant guidelines and regulations (Declaration of Helsinki). . Animal subjects: All authors have confirmed that this study did not involve animal subjects or tissue. Conflicts of interest: In compliance with the ICMJE uniform disclosure form, all authors declare the following: Payment/services info: All authors have declared that no financial support was received from any organization for the submitted work. Financial relationships: All authors have declared that they have no financial relationships at present or within the previous three years with any organizations that might have an interest in the submitted work. Other relationships: All authors have declared that there are no other relationships or activities that could appear to have influenced the submitted work.

\section{References}

1. Miura S, Miyata R, Matsumoto S, et al.: Quality management program of stroke rehabilitation using adherence to guidelines: a nationwide initiative in Japan . J Stroke Cerebrovasc Dis. 2019, 28:2434-41. 10.1016/j.jstrokecerebrovasdis.2019.06.028

2. The Japan Stroke Society: Japanese Guidelines for the Management of Stroke 2015 [Japanese]. The Japan Stroke Society Guideline Committee (ed): Kyowa Kikaku, Tokyo; 2015. https://www.kkkyowa.co.jp/stroke2015/.

3. Keith RA, Granger CV, Hamilton BB, Sherwin FS: The functional independence measure: a new tool for rehabilitation. Adv Clin Rehabil. 1987, 1:6-18.

4. Liu M, Domen K, Chino N: Comorbidity measures for stroke outcome research: a preliminary study . Arch Phys Med Rehabil. 1997, 78:166-172. 10.1016/s0003-9993(97)90259-8

5. Tsuji T, Liu M, Sonoda S, Domen K, Chino N: The stroke impairment assessment set: its internal consistency and predictive validity. Arch Phys Med Rehabil. 2000, 81:863-8. 10.1053/apmr.2000.6275

6. Inouye M: Predicting models of outcome stratified by age after first stroke rehabilitation in Japan . Am J Phys Med Rehabil. 2001, 80:586-91. 10.1097/00002060-200108000-00008

7. Sonoda S, Saitoh E, Nagai S, Okuyama Y, Suzuki T, Suzuki M: Stroke outcome prediction using reciprocal number of initial activities of daily living status. J Stroke Cerebrovasc Dis. 2005, 14:8-11.

10.1016/j.jstrokecerebrovasdis.2004.10.001

8. Tsuchiya K, Fujita T, Sato D, Midorikawa M, Makiyama Y, Shimoda K, Tozato F: Post-stroke depression inhibits improvement in activities of daily living in patients in a convalescent rehabilitation ward. J Phys Ther Sci. 2016, 28:2253-9. 10.1589/jpts.28.2253

9. Senda J, Ito K, Kotake T, et al.: Association of leukoaraiosis with convalescent rehabilitation outcome in patients with ischemic stroke. Stroke. 2016, 47:160-6. 10.1161/STROKEAHA.115.010682

10. Nishioka S, Wakabayashi H, Yoshida T, Mori N, Watanabe R, Nishioka E: Obese Japanese patients with stroke have higher functional recovery in convalescent rehabilitation wards: a retrospective cohort study. J Stroke Cerebrovasc Dis. 2016, 25:26-33. 10.1016/j.jstrokecerebrovasdis.2015.08.029

11. Shiraishi A, Yoshimura Y, Wakabayashi H, Tsuji Y: Poor oral status is associated with rehabilitation outcome in older people. Geriatr Gerontol Int. 2017, 17:598-604. 10.1111/ggi.12763

12. Matsushita T, Nishioka S, Taguchi S, Yamanouchi A: Sarcopenia as a predictor of activities of daily living capability in stroke patients undergoing rehabilitation. Geriatr Gerontol Int. 2019, 19:1124-8. 10.1111/ggi.13780

13. Nishioka S, Kokura Y, Okamoto T, Takayama M, Miyai I: Assignment of registered dietitians and other healthcare professionals positively affects weight change of underweight patients in convalescent (Kaifukuki) Rehabilitation wards: a secondary analysis of a nationwide survey. J Nutr Sci Vitaminol (Tokyo). 2019, 65:435-42. 10.3177/jnsv.65.435

14. Yoshimura Y, Wakabayashi H, Bise T, et al.: Sarcopenia is associated with worse recovery of physical function and dysphagia and a lower rate of home discharge in Japanese hospitalized adults undergoing convalescent rehabilitation. Nutrition. 2019, 61:111-8. 10.1016/j.nut.2018.11.005

15. Senda J, Ito K, Kotake T, et al.: Cilostazol use is associated with FIM cognitive improvement during convalescent rehabilitation in patients with ischemic stroke: a retrospective study [Japanese]. Nagoya J Med Sci. 2019, 81:359-73. 10.18999/nagjms.81.3.359

16. Tokunaga M, Sannomiya K, Nakanishi R, Yonemitsu H: The external validity of multiple regression analyses predicting discharge FIM score in patients with stroke hospitalized in Kaifukuki rehabilitation wards -An analysis of the Japan Rehabilitation Database [Japanese]. Jpn J Compr Rehabil Sci. 2015, 6:14-20. 10.11336/jicrs.6.14

17. Azimi P, Mohammadi HR, Benzel EC, Shahzadi S, Azhari S: Use of artificial neural networks to decision making in patients with lumbar spinal canal stenosis. J Neurosurg Sci. 2017, 61:603-11. 10.23736/S03905616.16.03078-2

18. Fujita T, Ohashi T, Yamane K, et al.: Relationship between the number of samples and the accuracy of the prediction model for dressing independence using artificial neural networks in stroke patients. Japanese J Compr Rehabil Sci. 2020, 11:28-34. 10.11336/jjcrs.11.28

19. Katsuki M, Kakizawa Y, Nishikawa A, Yamamoto Y, Uchiyama T: Easily created prediction model using deep learning software (Prediction One, Sony Network Communications Inc.) for subarachnoid hemorrhage outcomes from small dataset at admission. Surg Neurol Int. 2020, 11:374. 10.25259/SNI_636_2020

20. Katsuki M, Narita N, Ishida N, et al.: Preliminary development of a prediction model for daily stroke occurrences based on meteorological and calendar information using deep learning framework (Prediction 
One; Sony Network Communications Inc., Japan). Surg Neurol Int. 2021, 12:31. 10.25259/SNI_774_2020

21. Katsuki M, Narita N, Matsumori Y, Ishida N, Watanabe O, Cai S, Tominaga T: Preliminary development of a deep learning-based automated primary headache diagnosis model using Japanese natural language processing of medical questionnaire. Surg Neurol Int. 2020, 11:475. 10.25259/SNI_827_2020

22. Kumar R, Gupta A, Arora HS, Pandian GN, Raman B: CGHF: A computational decision support system for glioma classification using hybrid radiomics-and stationary wavelet-based features. IEEE Access. 2020, 8:79440-79458. 10.1109/ACCESS.2020.2989193

23. Fukuma R, Yanagisawa T, Kinoshita M, et al.: Prediction of IDH and TERT promoter mutations in low-grade glioma from magnetic resonance images using a convolutional neural network. Sci Rep. 2019, 9:20311. 10.1038/s41598-019-56767-3

24. Katsuki M, Kawamura S, Koh A: Easily created prediction model using automated artificial intelligence framework (Prediction One, Sony Network Communications Inc., Tokyo, Japan) for subarachnoid hemorrhage outcomes treated by coiling and delayed cerebral ischemia. Cureus. 2021, 13:e15695. 10.7759/cureus.15695

25. Watanabe O, Narita N, Katsuki M, Ishida N, Cai S, Otomo H, Yokota K: Prediction model of deep learning for ambulance transports in Kesennuma city by meteorological data. Open Access Emerg Med. 2021, 13:23-32. 10.2147/OAEM.S293551

26. Ignacio de Ulíbarri J, González-Madroño A, de Villar NG, et al.: CONUT: a tool for controlling nutritional status. first validation in a hospital population. Nutr Hosp. 2005, 20:38-45.

27. Katsuki M, Kakizawa Y, Nishikawa A, Yamamoto Y, Uchiyama T: Lower total protein and absence of neuronavigation are novel poor prognostic factors of endoscopic hematoma removal for intracerebral hemorrhage. J Stroke Cerebrovasc Dis. 2020, 29:105050. 10.1016/j.jstrokecerebrovasdis.2020.105050

28. Katsuki M, Kakizawa Y, Nishikawa A, Yamamoto Y, Uchiyama T: Endoscopic hematoma removal of supratentorial intracerebral hemorrhage under local anesthesia reduces operative time compared to craniotomy. Sci Rep. 2020, 10:10389. 10.1038/s41598-020-67456-x

29. Furtner J, Genbrugge E, Gorlia T, et al.: Temporal muscle thickness is an independent prognostic marker in patients with progressive glioblastoma: translational imaging analysis of the EORTC 26101 trial. Neuro Oncol. 2019, 21:1587-94. 10.1093/neuonc/noz131

30. Steindl A, Leitner J, Schwarz M, et al.: Sarcopenia in neurological patients: standard values for temporal muscle thickness and muscle strength evaluation. J Clin Med. 2020, 9:1272. 10.3390/jcm9051272

31. Furtner J, Berghoff AS, Schöpf V, et al.: Temporal muscle thickness is an independent prognostic marker in melanoma patients with newly diagnosed brain metastases. J Neurooncol. 2018, 140:173-8. 10.1007/s11060018-2948-8

32. Katsuki M, Yamamoto Y, Uchiyama T, Wada N, Kakizawa Y: Clinical characteristics of aneurysmal subarachnoid hemorrhage in the elderly over 75 ; would temporal muscle be a potential prognostic factor as an indicator of sarcopenia?. Clin Neurol Neurosurg. 2019, 186:105535. 10.1016/j.clineuro.2019.105535

33. Katsuki M, Suzuki Y, Kunitoki K, et al.: Temporal muscle as an indicator of sarcopenia is independently associated with Hunt and Kosnik grade on admission and the modified Rankin Scale at 6 month of patients with subarachnoid hemorrhage treated by endovascular coiling. World Neurosurg. 2020, 137:e526-34. 10.1016/j.wneu.2020.02.033

34. Furtner J, Berghoff AS, Albtoush OM, et al.: Survival prediction using temporal muscle thickness measurements on cranial magnetic resonance images in patients with newly diagnosed brain metastases. Eur Radiol. 2017, 27:3167-73. 10.1007/s00330-016-4707-6

35. Kaifukuki Rehabilitation Ward Association: Survey report on the current status and issues of rehabilitation wards for persons in kaifukuki rehabilitation wards [Japanese]. Kaifukuki Rehabilitation Ward Association (ed): Tokyo; 2020.

36. Peduzzi P, Concato J, Kemper E, Holforf TR, Feinstein AR: A simulation study of the number of events per variable in logistic regression analysis. J Clin Epidemiol. 1996, 49:1373-1379. 10.1016/S0895-4356(96)002363

37. Maki S, Furuya T, Yoshii T, et al.: Machine learning approach in predicting clinically significant improvements after surgery in patients with cervical ossification of the posterior longitudinal ligament. Spine (Phila Pa 1976). 2021, 10.1097/BRS.0000000000004125 